\title{
Starvation promotes odor/feeding-time associations in flies
}

\author{
Nitin Singh Chouhan, Reinhard Wolf, and Martin Heisenberg \\ Rudolf-Virchow-Center, 97080 Würzburg, Germany
}

\begin{abstract}
Starvation causes a motivational state that facilitates diverse behaviors such as feeding, walking, and search. Starved Drosophila can form odor/feeding-time associations but the role of starvation in encoding of "time" is poorly understood. Here we show that the extent of starvation is correlated with the fly's ability to establish odor/feeding-time memories. Prolonged starvation promotes odor/feeding-time associations after just a single cycle of reciprocal training. We also show that starvation is required for acquisition but is dispensable for retrieval of odor/feeding-time memory. Finally, even with extended starvation, a functional circadian oscillator is indispensable for establishing odor/feeding-time memories.
\end{abstract}

[Supplemental material is available for this article.]

Many processes in the living world occur at a certain time in the daily cycle and organisms have a circadian clock that helps organizing their behavior accordingly. One such service of the clock is to provide the phase information to form time-specific memories. This may reduce energy expenditures in finding essential resources. For instance, mates gathering at a certain place or food associated with some odor might be available only at a certain time of day. With a time and place or odor association, the mates and food would be more easily retrieved. The importance of such timespecific associations is highlighted by the fact that most animals tested make use of it (Gould 1987; Harrison and Breed 1987; Biebach et al. 1989; Boulos and Logothetis 1990; Zhang et al. 2006; Van der Zee et al. 2008; Chouhan et al. 2015).

Earlier studies revealed that indeed the circadian clock is involved (Biebach et al. 1991; Wenger et al. 1991; Mistlberger et al. 1996; Van der Zee et al. 2008; Chouhan et al. 2015). Moreover, the ability to establish time-specific association depends upon certain internal states. Starved but not satiated Wistar rats show an association of "time" with the location of the platform in a Morris water maze (Lukoyanov et al. 2002). Similar studies with Sprague Dawley rats also showed that well-fed animals performed timeplace memory only when their "response cost" was increased through added weight belts (Widman et al. 2004). Low cost for switching between different choices in a memory test has been assumed to result in poor time-place associations, not only in rats but also in cichlid fishes (Boulos and Logothetis 1990; Reebs 1993). Accordingly, food deprivation is thought to increase the response cost and therefore promote time-specific associations (Widman et al. 2000, 2004). These studies highlight the importance of the internal state of an animal in facilitating time-of-day memory formation but our understanding of this process and its control remains elusive.

Here we show in Drosophila melanogaster that survival rate under chronic starvation is correlated with odor/feeding-time memory (OFM) (Chouhan et al. 2015). We propose that severe starvation increases the cost associated with the wrong decisions in the search for food and that this state of malnutrition induces flies to use "time" as an associative cue.

In Drosophila odor/feeding-time memory is based on appetitive long-term memory (ALM) (Krashes and Waddell 2008). In the

Corresponding author: heisenberg@biozentrum.uni-wuerzburg.de Article is online at http://www.learnmem.org/cgi/doi/10.1101/lm.045039.117. appetitive long-term memory assay used here, flies starved for $18 \mathrm{~h}$

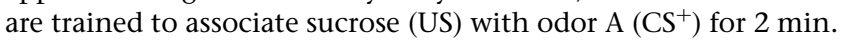
After a stream of clean air for $30 \mathrm{sec}$, flies are presented with a watersoaked filter paper plus odor $\mathrm{B}\left(\mathrm{CS}^{-}\right)$for $2 \mathrm{~min}$, followed by another $30 \mathrm{sec}$ stream of clean air. Memory is tested after $24 \mathrm{~h}$ by presenting flies in a T-maze with odor A and odor B for $2 \mathrm{~min}$. In the present study 4-methylcyclohexanol and 3-octanol are used as odors.

For the odor/feeding-time memory assay, two groups of about 150-200 flies are starved for $14 \mathrm{~h}$ and then presented with odor A together with sucrose and odor B without sucrose in the morning in a T-maze. After training flies are returned to the vials with no food. The same flies are given sucrose with odor B and a water-soaked filter paper with odor A in the afternoon, after $20 \mathrm{~h}$ of starvation. Subsequently they are fed for an hour on regular food to keep them alive (emergency feeding). This training cycle is repeated on the second day at which point flies are already starved for $38 / 44 \mathrm{~h}$ (disregarding the $1 \mathrm{~h}$ emergency feeding). On the third day, one group of flies is tested for memory in a T-maze apparatus in the morning and the other group in the afternoon. Flies prefer odor A in the morning and then significantly change their preference to odor B in the afternoon (Fig. 1B; Chouhan et al. 2015). Evidently, flies can use the time of day as an associative cue but the experiments do not highlight the role of starvation in establishing odor/feeding-time memories.

Rats show significantly better memory retention after onetrial active or passive avoidance conditioning when tested at the same time of day as the training (Holloway and Wansley 1973a,b). Thus, animals may automatically encode the time of day information with a biologically significant event (Gallistel 1990).

In contrast, flies do not show such periodic modulation in the memory retrieval. Eighteen-hour-starved flies were trained in the appetitive long-term memory paradigm at ZTO (Zeitgeber Time; ZTO refers to the time of "lights-on" and ZT12 to "lights-off) or ZT6 and then tested the next day in the morning or in the afternoon. We observed no significant difference in the appetitive

\footnotetext{
(C) 2017 Chouhan et al. This article is distributed exclusively by Cold Spring Harbor Laboratory Press for the first 12 months after the full-issue publication date (see http://learnmem.cshlp.org/site/misc/terms.xhtml). After 12 months, it is available under a Creative Commons License (AttributionNonCommercial 4.0 International), as described at http://creativecommons. org/licenses/by-nc/4.0/.
} 
A

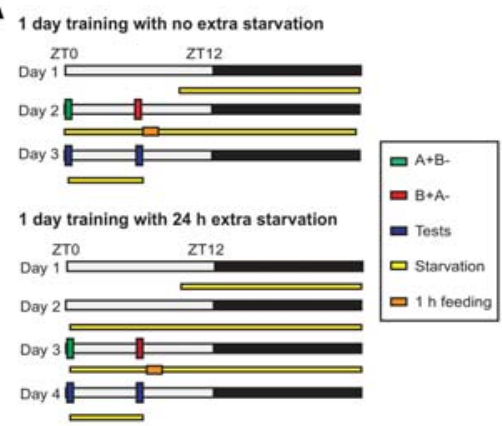

C

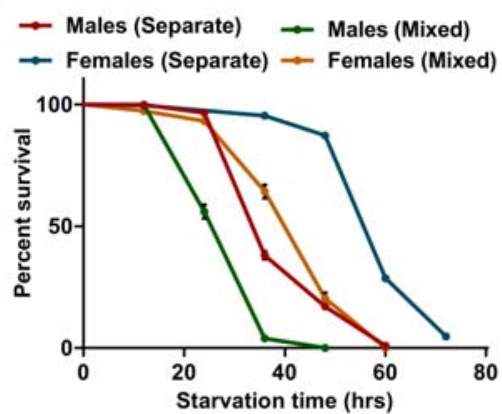

E

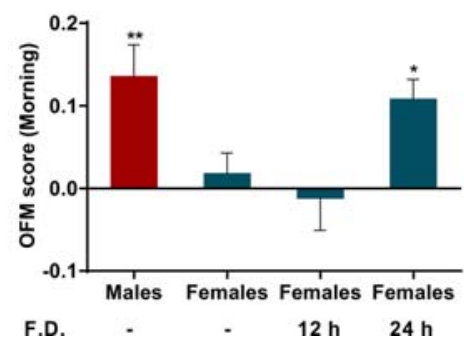

B

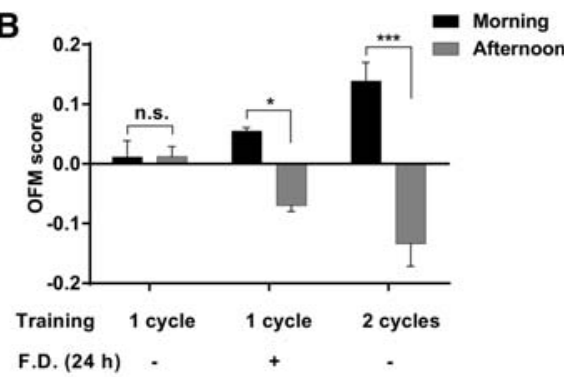

D

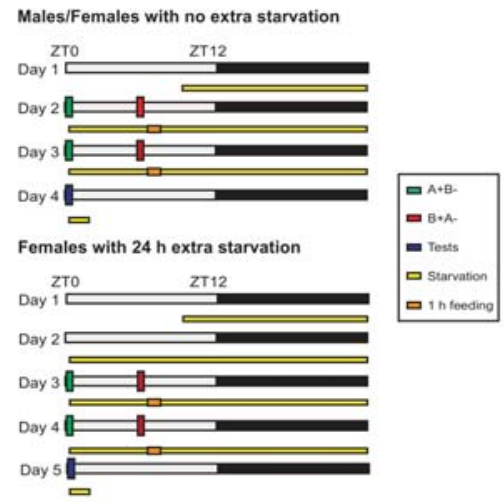

single cycle of reciprocal training sessions. Flies starved for $14 \mathrm{~h}$, trained with a single cycle and tested the next day (Fig. 1A) showed no significant odor/feeding-time memory in morning and afternoon tests (Fig. 1B). The role of prolonged food deprivation in odor/ feeding-time memory formation was then examined by starving wild-type flies (WT) of the strain Canton-S for an extra $24 \mathrm{~h}$ and then training them with a single cycle (Fig. 1A). Now they showed robust odor/feeding-time memory scores related to morning and afternoon training sessions (Fig. 1B). With $38 / 44 \mathrm{~h}$ of starvation, flies trained with two cycles demonstrated significantly better odor/ feeding-time memory scores than flies trained with only a single cycle (Fig. 1B). These results suggest that extended starvation can promote formation of time-odor memory. Also, training the flies after $38 / 44 \mathrm{~h}$ starvation with a single cycle of reciprocal trials is sufficient to form odor/feeding-time memories.

If severe starvation increases the cost associated with the wrong decisions in the search for food, the starvation periods promoting odor/feeding-time memory might already show effects in the survival rate. We therefore measured the effects of food deprivation on survival. For these experiments eight vials, each of which contained about 100 flies, were used for every experimental group. Flies were placed in vials, with no food, containing only a thin layer of $1 \%$ agarose. These flies were kept in standard conditions and the numbers of surviving flies were counted every $12 \mathrm{~h}$. Survival rate at each time point was calculated as the number of surviving flies divided by the total number of flies (Fig. 1C; Kaplan-Meier survival curves).

Measuring survival, we noted a difference between males and females. We decided to also raise and test male and female flies separately. This prolonged survival in both genders. Isolated females survived about $24 \mathrm{~h}$ longer than isolated males (Fig. 1C).

As with the starvation periods chosen significant numbers of flies were

long-term memory retrieval between flies tested at ZT0 and ZT6 (Supplemental Fig. 1). Also, the ability of flies to perform in an appetitive-conditioning paradigm was comparable between ZT0 and ZT6, which indicates that acquisition and retrieval of appetitive long-term memory in flies is not affected by the time of day (Supplemental Fig. 1). These results imply that flies do not associate "time" with every appetitive training. This raises the question about how flies develop odor/feeding-time memory and whether the degree of starvation influences such associations.

Chouhan et al. (2015) had demonstrated that two cycles of training and $38 / 44 \mathrm{~h}$ of starvation are sufficient to facilitate time-odor learning. We reconfirmed this result (Fig. 1B) and asked whether flies can form time-odor associations also after a dead, this precluded the afternoon memory test. Regarding the morning test, males, but not females showed robust odor/ feeding-time memory related to the morning training (Fig. $1 \mathrm{D}, \mathrm{E})$. In contrast, in the appetitive long-term memory assay male and female flies showed comparable scores (Supplemental Fig. 2). This outcome suggested that female flies' low performance in odor/feeding-time memory was not due to their compromised ability to form appetitive memory but rather hinted at a correlation between survival rate and the ability to form odor/feedingtime memory. In isolated males $14 / 20 \mathrm{~h}$ of starvation had no substantial effects on survival, whereas $38 / 44 \mathrm{~h}$ of starvation significantly diminished the survival rate. Therefore, these results suggested that the second cycle of training during which males 
A

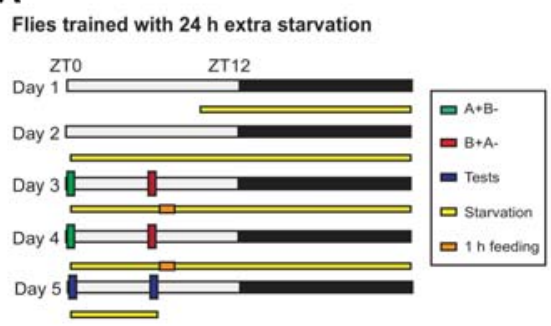

B

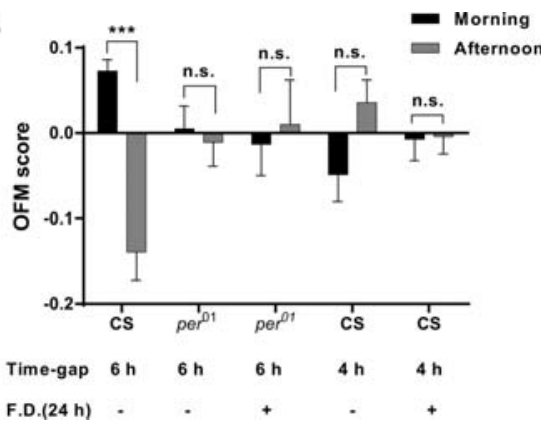

Figure 2. Starvation induced odor/feeding-time association requires a functional clock and a minimal time-gap. (A) Schematic describing time-odor learning experiments in flies with $24 \mathrm{~h}$ extra food deprivation before the training. $(B) p r^{01}$ mutants were unable to display time-specific memories even after prolonged starvation. Added periods of starvation failed to induce significant odor/feeding-time memory in WT flies that were trained with a time-gap of $4 \mathrm{~h}$ between morning and afternoon conditioning cycles $(n \geq 6) ;\left({ }^{* *}\right) P<0.001$; (FD) extra food deprivation.

were under higher starvation stress promoted odor/feeding-time associations. Also, male flies were under more starvation stress than female flies, as shown by the lower survival rate in response to food deprivation (Fig. 1C). This may have promoted odor/ feeding-time memory in males but not in females.

Consequently, increased starvation stress might promote time-specific memory formation also in isolated females. To examine this hypothesis female CS flies were subjected to additional periods of starvation beyond the $14 / 20 \mathrm{~h}$ of the standard protocol (Fig. 1D). An extra $24 \mathrm{~h}$ of food deprivation before training will result in $38 / 44 \mathrm{~h}$ and $62 / 68 \mathrm{~h}$ starvation during first and second cycles of training, respectively. Female flies that were starved for extra $24 \mathrm{~h}$ before the training showed significant odor/feeding-time memory when tested in the morning after two cycles of time-odor conditioning (Fig. 1E). This result suggests that higher resistance to starvation might be responsible for the low time-odor learning performance in female flies. After $62 \mathrm{~h}$ females are under greater starvation stress during the second cycle of training, as shown by the lower survival rate, which may promote odor/feeding-time memory.

Survival of flies in a mixed population was significantly lower than flies raised separately (Fig. 1C). This indicates that flies are under higher starvation stress in a mixed group, shown by lower survival rate after $38 / 44 \mathrm{~h}$ starvation, which may promote formation of odor/ feeding-time memory after just a single training cycle (Fig. 1B). All these results together support the hypothesis that response cost based on food deprivation matters for odor/feeding-time memory.

Circadian clock mutants, per $^{01}$ and $c l k^{A R}$, are unable to perform in the time-odor learning paradigm (Chouhan et al. 2015). We tested if prolonging the starvation can enable per ${ }^{01}$ mutant flies to associate the time of day with appeti-

a lower time-gap between reciprocal trials.

tive memories. per ${ }^{01}$ mutants showed no significant morning or afternoon odor/ feeding-time memory scores even with $24 \mathrm{~h}$ additional starvation before the training (Fig. 2A,B).

Previous work had shown that flies need at least a 6-h interval between reciprocal trials to establish odor/feedingtime memory (Chouhan et al. 2015). We examined whether flies were able to remember "time" events separated by 4 $\mathrm{h}$ after added $24 \mathrm{~h}$ starvation. We observed no significant odor/feeding-time memory scores when WT flies were trained at ZT0 (morning) and ZT4 (afternoon) to associate reward and odor (Fig. 2B). These results suggest that prolonging starvation in flies is not sufficient to promote time-specific memory formation without a functional clock or with Acute starvation before training helps flies differentiate reciprocal trials according to the time of day at which they occurred. We next asked if such prolonged starvation is necessary only before memory formation or is also required for odor/feeding-time memory retrieval. To investigate the temporal requirement of
A

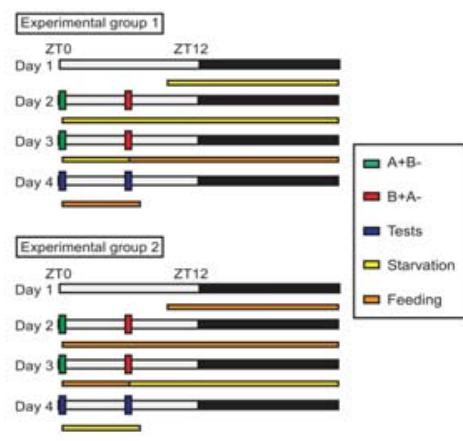

C

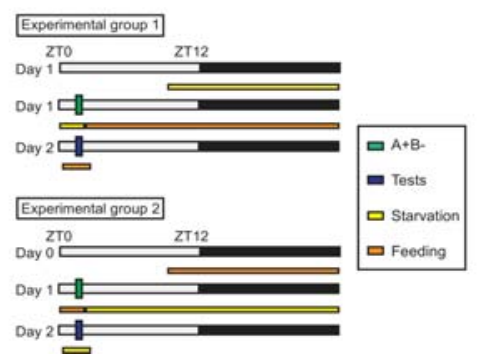

B

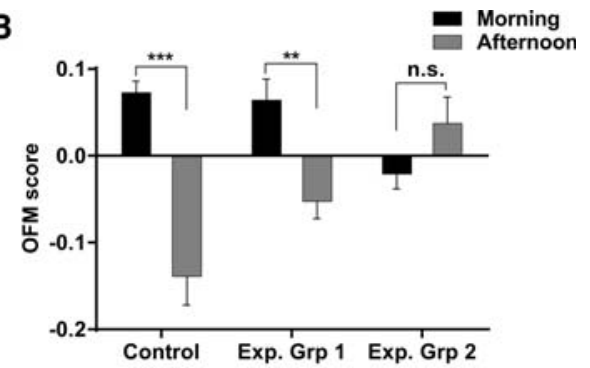

D

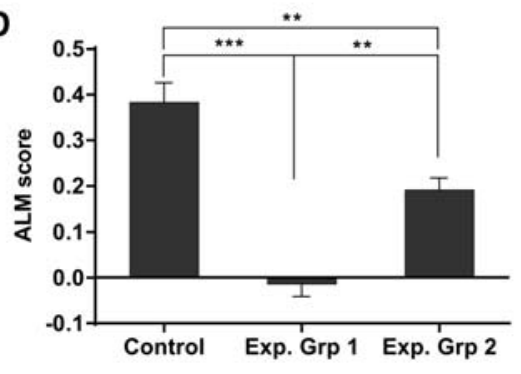

Figure 3. Food deprivation before and during training is required for odor/feeding-time memory formation. (A) Schematic describing different starvation schedules for flies trained in time-odor learning paradigm. Exp. Grp. 1 flies were subjected to starvation before and during conditioning but then moved to food vials while flies in Exp. Grp 2 were kept on food but then moved to agar vials after the last conditioning event. (B) Odor/feeding-time memory: Flies starved before and during conditioning (Exp. Grp 1) demonstrate robust odor/feeding-time memory scores (OFM) in morning and afternoon tests. In contrast, flies starved after the last training event (Exp. Grp 2) did not. $(n \geq 8) ;\left(^{*}\right) P<$ $\left.0.05 ;{ }^{* *}\right) P<0.01 ;(* * *) P<0.001$. (C) Appetitive long-term memory experiments with different starvation schedules. Exp. Grp. 1 flies were kept on food vials after conditioning while Exp. Grp. 2 flies were starved after conditioning. (D) Appetitive long-term memory: Flies starved before and during conditioning but subsequently transferred to food vials (Exp. Grp 1) did not show significant performance. Flies that were only starved after training (Exp. Grp 2) demonstrated a significant appetitive long-term memory score (ALM) but were considerably compromised compared with WT flies that were starved throughout the experiment $(n=6) ;(* *) P<0.01 ;\left({ }^{* * *}\right) P<0.001$. 
starvation for time-specific memories, one group of CS flies (Exp. Grp 1) was subjected to starvation during conditioning but then moved to food vials after the last training and was tested the next morning and afternoon (Fig. 3A). Another group of flies (Exp. Grp 2) was kept on food before conditioning but was moved to agar vials after the last conditioning event and then tested on the third day (Fig. 3A). Before each training cycle, the fed flies were placed in agar vials for $30 \mathrm{~min}$ to stimulate ingestion of sucrose during the training. The control flies were kept under food deprivation for all days of the experiment. Flies that were starved only during the training but not before testing performed as well as control flies in forming odor/feeding-time memory (Fig. 3B). These results indicate that starvation is essential for memory formation but may be dispensable for odor/feeding-time memory retrieval.

These findings are at odds with previous studies on appetitive long-term memory, which showed that food deprivation is required for retrieval of the memory (Krashes and Waddell 2008; Krashes et al. 2009; Liu et al. 2012). As these experiments were conducted with a single training cycle, we conducted appetitive longterm memory experiments with different feeding schedules. Exp. Grp 1 of WT flies was starved before training but then moved to food vials and flies in Exp. Grp 2 were only starved after conditioning (Fig. 3C). Control flies were kept on agar vials for the experiment. As observed before, flies that were satiated before testing were unable to show significant appetitive long-term memory score (Fig. 3D; Krashes and Waddell 2008; Liu et al. 2012). In contrast, flies that were starved after conditioning showed a robust appetitive long-term memory score even if their PI was significantly lower than in the control group flies (Fig. 3D). Therefore, in contrast to odor/feeding-time memory, starvation before testing is required for appetitive long-term memory retrieval.

Here we have demonstrated the requirement of acute starvation for promoting the formation of odor/feeding-time associations in flies. Female WT flies that can stand prolonged starvation compared with males, require an extra period of food deprivation to establish odor/feeding-time memory (Fig. 1D,E). This holds for mixed populations as well as isolated female and male groups. The better performance in odor/feeding-time memory in mixed populations correlates with higher starvation stress (Fig. 1C). The survival rate of starved females in mixed populations is higher than that of the males and social interactions seem to increase starvation stress for females as well as males (Fig. 1C). These observations raise the question about a special role of social interactions in forming odor/feeding-time memories. Previous studies have highlighted the role of social interactions in modulating the survival rate and memory retrieval in flies (Ruan and $\mathrm{Wu}$ 2008; Chabaud et al. 2009; Bretman et al. 2013). The present findings rather suggest that the social interactions affect the promotion of odor/feeding-time memory via starvation stress.

Our results also show that the second cycle of training promotes odor/feeding-time memory in addition to the effect of the extended starvation period. On the other hand, given that a single cycle of training is sufficient, the repetition of training cycles is not an absolute requirement for the formation of time-of-day-related memories (Fig. 1A,B).

The results suggest that acute starvation increases the "response cost" in flies, as wrong choices or decisions may prevent food discovery. Severe starvation activates a mechanism, molecular and neuronal, that links a time-of-day signal to another signal promising food. This time-of-day information requires a functional circadian clock as per ${ }^{01}$ mutant flies are unable to perform even after added periods of starvation before the training (Fig. 2B). Results also show that starvation only during training is sufficient to promote odor/feeding-time associations (Fig. 3). This suggests that it is the formation of time-related odor memories that is cost-intensive. Retrieval works even in satiated flies, pointing to- ward the intriguing possibility that the retrieval mechanism of odor/feeding-time memory differs from that of appetitive longterm memory. The mechanistic understanding of how starvation controls the formation and storage of circadian clock based odor/ feeding-time memories awaits future studies.

\section{Acknowledgments}

We thank A. Haberberger for technical assistance; the Department of Neurobiology and Genetics, Biozentrum Wuerzburg for support in fly maintenance; and the mechanics workshop (H. Kaderschabek) and the electronics workshop (K. Oechsner) of the Biozentrum for technical support. The project was funded by the German Science Foundation (SFB 1047; Insect Timing and Reinhard Koselleck Project, He986/20-1).

\section{References}

Biebach H, Gordijn M, Krebs JR. 1989. Time and place learning by garden warblers, Sylvia borin. Anim Behav 37: 353-360.

Biebach H, Falk H, Krebs JR. 1991. The effect of constant light and phase shifts on a learned time-place association in garden warblers (Sylvia borin): hourglass or circadian clock? J Biol Rhythms 6: 353-365.

Boulos Z, Logothetis DE. 1990. Rats anticipate and discriminate between two daily feeding times. Physiol Behav 48: 523-529.

Bretman A, Westmancoat JD, Gage MJ, Chapman T. 2013. Costs and benefits of lifetime exposure to mating rivals in male Drosophila melanogaster. Evolution 67: 2413-2422.

Chabaud M-A, Isabel G, Kaiser L, Preat T. 2009. Social facilitation of long-lasting memory retrieval in Drosophila. Curr Biol 19: 1654-1659.

Chouhan NS, Wolf R, Helfrich-Förster C, Heisenberg M. 2015. Flies remember the time of day. Curr Biol 25: 1619-1624.

Gallistel CR. 1990. Representations in animal cognition: an introduction. Cognition 37: 1-22.

Gould JL. 1987. Honey bees store learned flower landing behavior according to time of day. Anim Behav 35: 1579-1581.

Harrison JM, Breed MD. 1987. Temporal learning in the giant tropical ant, Paraponera clavata. Physiol Entomol 12: 317-320.

Holloway FA, Wansley R. 1973a. Multiphasic retention deficits at periodic intervals after passive-avoidance learning. Science 180: 208-210.

Holloway FA, Wansley RA. 1973b. Multiple retention deficits at periodic intervals after active and passive-avoidance learning. Behav Biol 9: 1-14.

Krashes MJ, Waddell S. 2008. Rapid consolidation to a radish and protein synthesis-dependent long-term memory after single-session appetitive olfactory conditioning in Drosophila. J Neurosci 28: 3103-3113.

Krashes MJ, DasGupta S, Vreede A, White B, Armstrong JD, Waddell S. 2009. A neural circuit mechanism integrating motivational state with memory expression in Drosophila. Cell 139: 416-427.

Liu C, Plaçais PY, Yamagata N, Pfeiffer BD, Aso Y, Friedrich AB, Siwanowicz I, Rubin GM, Preat T, Tanimoto H. 2012. A subset of dopamine neurons signals reward for odour memory in Drosophila. Nature 488: 512-516.

Lukoyanov NV, Pereira PA, Mesquita RM, Andrade JP. 2002. Restricted feeding facilitates time-place learning in adult rats. Behav Brain Res 134: 283-290.

Mistlberger RE, de Groot MH, Bossert JM, Marchant EG. 1996. Discrimination of circadian phase in intact and suprachiasmatic nuclei-ablated rats. Brain Res 739: 12-18.

Reebs SG. 1993. A test of time-place learning in a cichlid fish. Behav Processes 30: $273-281$.

Ruan H, Wu C-F. 2008. Social interaction-mediated lifespan extension of Drosophila $\mathrm{Cu} / \mathrm{Zn}$ superoxide dismutase mutants. Proc Natl Acad Sci 105: $7506-7510$.

Van der Zee EA, Havekes R, Barf RP, Hut RA, Nijholt IM, Jacobs EH, Gerkema MP. 2008. Circadian time-place learning in mice depends on Cry genes. Curr Biol 18: 844-848.

Wenger D, Biebach H, Krebs JR. 1991. Free-running circadian rhythm of a learned feeding pattern in starlings. Naturwissenschaften 78: 87-89.

Widman DR, Gordon D, Timberlake W. 2000. Response cost and time-place discrimination by rats in maze tasks. Anim Learn Behav 28: 298-309.

Widman DR, Sermania CM, Genismore KE. 2004. Evidence for time-place learning in the Morris water maze without food restriction but with increased response cost. Behav Process 67: 183-193.

Zhang SW, Schwarz S, Pahl M, Zhu H, Tautz J. 2006. Honeybee memory: a honeybee knows what to do and when. J Exp Biol 209: 4420-4428.

Received January 18, 2017; accepted in revised form April 25, 2017. 


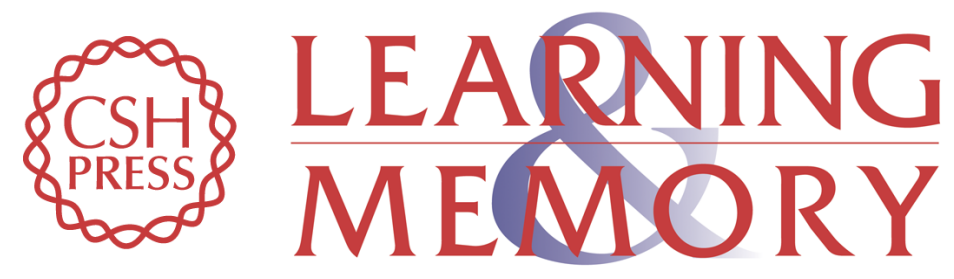

\section{Starvation promotes odor/feeding-time associations in flies}

Nitin Singh Chouhan, Reinhard Wolf and Martin Heisenberg

Learn. Mem. 2017, 24:

Access the most recent version at doi:10.1101/lm.045039.117

Supplemental http://learnmem.cshlp.org/content/suppl/2017/06/07/24.7.318.DC1
Material

References This article cites 23 articles, 4 of which can be accessed free at: http://learnmem.cshlp.org/content/24/7/318.full.html\#ref-list-1

Creative This article is distributed exclusively by Cold Spring Harbor Laboratory Press for the Commons first 12 months after the full-issue publication date (see

License http://learnmem.cshlp.org/site/misc/terms.xhtml). After 12 months, it is available under a Creative Commons License (Attribution-NonCommercial 4.0 International), as described at http://creativecommons.org/licenses/by-nc/4.0/.

Email Alerting Receive free email alerts when new articles cite this article - sign up in the box at the Service top right corner of the article or click here. 